\title{
U.S. Dept. Veterans Affairs (VA) SMEC-bio Reporting for Leadership Decision Support
}

\author{
Shantini D. Gamage ${ }^{\star 1,2}$, Loretta A. Simbartl ${ }^{1}$, Stephen M. Kralovic ${ }^{1,2,3}$, Katherine S. \\ Wallace ${ }^{4}$ and Gary A. Roselle ${ }^{1,2,3}$
}

'National Infectious Diseases Service, Veterans Health Administration (VHA), Washington, DC, USA; 2University of Cincinnati College of Medicine, Cincinnati, OH, USA; ${ }^{3} \mathrm{Cincinnati}$ VA Medical Center, Cincinnati, OH, USA; ${ }^{4} \mathrm{Office}$ of Operations, Security and Preparedness (OSP), VA Central Office, Washington, DC, USA

\section{Objective}

To assess Reports sent from the United States VA Subject Matter Expertise Center for Biological Events (SMEC-bio) - a proof-ofconcept decision support initiative - to the VA Integrated Operations Center (VA IOC)

\section{Introduction}

VA is the U.S. federal agency responsible for providing services to America's Veterans. Within VA, VHA is the organization responsible for administration of health care services. VHA, with 152 Medical Centers and over 900 outpatient clinics located throughout the U.S and territories, provided care to over 5 million patients in 2011.

After the $2009 \mathrm{H} 1 \mathrm{~N} 1$ influenza pandemic, OSP, which oversees VA senior level briefing of preparedness issues, conceptualized and initiated SMEC-bio as a protocol-based mechanism to incorporate timely VHA subject matter expertise into leadership decision making via the VA IOC. Previous work has examined collection and integration of data from VA and interagency sources for trend and predictive analyses (1). This current work is an initial assessment of SMEC-bio reporting, which has been in development for the past year and functions on an ad hoc basis for decision support; needs and gaps can be assessed toward a formalized communication plan with the VA IOC.

\section{Methods}

In May, 2011, SMEC-bio designed a Report template. All SMECbio Reports submitted to the VA IOC using the template were assessed based on reason for the Report, timing, data sources used, and outcome. A gap analysis was conducted to identify areas for further improvement

\section{Results}

Eight SMEC-bio Reports were produced since the template was initiated in May, 2011. The reasons for reporting fell into the following categories: 1) briefings of interagency protocol activations [e.g. National Biosurveillance Integration System (NBIS) protocol]; 2) Requests for Information (RFIs) from the VA IOC regarding specific biological events (e.g. $2012 \mathrm{H} 3 \mathrm{~N} 2 \mathrm{v}$ influenza associated with swine at fairs); 3) RFIs from the VA IOC on general infectious diseases issues (e.g. 2011 dust storm in Arizona); and 4) SMEC-bio-initiated Reports to provide situational awareness to the VA IOC on a biological event (e.g. the measles outbreak at the time of the 2012 Super Bowl in Indiana).

Reports in response to RFIs were all submitted within the day, often within hours including those that required data collection and interpretation, indicating that SMEC-bio can be a viable source for timely decision support to senior leadership. Some Reports, such as the one on possible infectious diseases issues after hurricane Irene in August, 2011, were subsequently shared by VA IOC with VHA Operations and with field facilities, thus highlighting the potential for facilitating provision of timely subject matter expertise for local response.

The primary information source for Reports was the Centers for Disease Control and Prevention website, press releases, and interagency briefings. Data sources used were the VHA centrally-administered electronic health records system and syndromic surveillance via VA-adapted ESSENCE.

Gap analysis results included common themes for biopreparedness: uncertainty in data quality and interpretation, communication of results and confidence levels to leadership, and coordination among stakeholders. Furthermore, the development of a decision tool to guide selection of events for reporting will be a critical initial requirement of a formal communications plan.

\section{Conclusions}

As SMEC-bio progresses from proof-of-concept phase to development status, knowledge gained from ad hoc reporting, as described in this work, will be critical for developing a routine and effective communications plan. Other ongoing work that will support communications include staffing assessments, development of analysis tools, and incorporating automated report capabilities.

\section{Keywords}

Reporting; Decision Support; Communications

\section{Acknowledgments}

The authors thank JC Cantrell, VA IOC Director, and VA IOC staff for helpful interactions regarding reporting.

\section{References}

1. Wallace, KS, et al. U.S. Department of Veterans Affairs Integrated Operations Center (VA IOC): Collaborations for Surveillance, Analysis, and Prediction for Infectious Disease Threat Preparedness - Pilot Review of Dengue Occurrence. Emerg. Health Threats J. 4:s155, 2011

*Shantini D. Gamage

E-mail: shantini.gamage@va.gov 